\title{
Similarity transformation in PT-symmetry: limitations
}

\begin{abstract}
We show that proper use of similarity transformation in PT-symmetry operator can justify real spectrum. Further we discuss its limitation. As an example of this we consider harmonic oscillator to demonstrate this. PACS no: 03.65.G; 11.30.Pb

Keywords: spectral reality, harmonic oscillator, similarity transformation, PTsymmetry
\end{abstract}

Volume I Issue 4 - 2018

Biswanath Rath

Department of Physics, North Orissa University, India

Correspondence: Biswanath Rath, Department of Physics, North Orissa University, Takatpur, Baripada-757003, Odisha, India, Email biswanathrathI0@gmail.com

Received: July 26, 2018| Published: August 21, 2018

\section{Introduction}

Fundamental postulates of quantum mechanics are based on selfad joint operator.

$$
H=H^{\dagger}
$$

Almost all the text books discuss real spectra relating to self adjoint operator. ${ }^{1}$ However contrary to this common understanding, Bender and Boettecher ${ }^{2}$ have introduced $\mathcal{P} \mathcal{T}$-symmetry. Here $\mathcal{P}$-stands for parity operator having behavior of space reflection: $\mathcal{P}(x, y, z) \rightarrow(-x,-y,-z)$

and $\mathcal{T}$-stands for time reversal operator, having behavior of time reversal: $\mathcal{T}(i) \rightarrow I$. In fact under $\mathcal{P} \mathcal{T}$-operator the commutation relation.

$$
[x, p]=i
$$

Remains invariant. In order to co-relate real spectra with $\mathcal{P} \mathcal{T}$ operator, Mostafazadeh ${ }^{3,4}$ proposed the word pseudo-hermitisity and introduced the condition

$$
\Omega H_{\mathcal{P} \mathcal{T}} \Omega^{-1}=h=h^{\dagger}
$$

According to author ${ }^{2}$ all $\mathcal{P} \mathcal{T}$-invariant operators satisfying above condition must possess real iso-spectra with the parent operator. ${ }^{3-6}$ In other words real iso-spectra nature exists between $H$ and $h$. The condition proposed in $\mathrm{Eq}(2)$ is that $\Omega$ must be a bounded one. In order to justify that author ${ }^{3,4}$ showed that unbound operator $\Omega$ will lead non-iso-spectral behaviour. ${ }^{3,4}$ The example proposed by the author ${ }^{3,4}$ is as follows

$$
\begin{aligned}
& \Omega_{1}=e^{ \pm \frac{x^{3}}{3}} \\
& \Omega_{1}^{-1}\left[h=p^{2}+x^{4}\right] \Omega_{4}=p^{2} \pm i\left(x^{2} p+p x^{2}\right)
\end{aligned}
$$

In fact author's justication in above example to stress the bounded nature on $\Omega$ is justified.,

On the other hand contrary to above justification, we feel only suitably or properly selected bounded operator will yield only real spectra. In fact if $\Omega$ is not properly selected then one may lose the isospectral nature between $H_{\mathcal{P} \mathcal{T}}$ and $h^{\dagger}$. In other words similarity transformation has certain limitations. However beginners or first readers in quantum mechanics may not accept it so easily. In order to convince the all interested readers, we present few examples relating to harmonic oscillator. The purpose of considering harmonic oscillator is that its wave function and eigenvalues are exactly known [I], the details are given below.

\section{Similarity \\ transformation and spectral invariance}

Consider an operator having real eigenvalues as given below

$$
H \psi>=E \psi>
$$

Let us consider another operator having the same eigenvalue as

$h \Phi>=\in \Phi>$

Then

And

$$
\sum_{m}\left|\psi_{m}><\psi_{m}\right|=\sum_{m}\left|\Phi_{m}><\Phi_{m}\right|=I
$$

$$
<\psi_{m}|\mathrm{H}| \psi_{m}>=E_{m}=<\psi_{m} S^{-1}\left(S H S^{-1}\right) S \psi_{m}>=<\Phi_{m}|h| \Phi_{m}>
$$

If $E_{m}=\epsilon_{m}$ then we have

$$
\sum_{m} E_{m}\left|\psi_{m}><\psi_{m}\right|=\sum_{m} \in_{m}\left|\Phi_{m}><\Phi_{m}\right|
$$

Implies the following

$$
\begin{aligned}
& S H S^{-1}=h \Leftrightarrow S^{-1} h S=H \\
& \Phi_{m}>=S \mid \psi_{m}>
\end{aligned}
$$

And

$$
<\psi_{m}\left\|\psi_{m}>=<\Phi_{m}\right\| \Phi_{m}>=1
$$

In the case of bounded operator $S^{-1} \neq S^{\dagger}$ even then normalization is conserved.

$$
<\Phi_{m}\left|=<\psi_{m}\right| S^{-1}
$$

This helps us to define few relations as

$$
\begin{gathered}
h=\sum_{m} E_{m}\left|\Phi_{m}><\Phi_{m}\right|=\sum_{m} E_{m}\left|\Phi_{m}><\psi_{m}\right| \mathrm{S}^{-1} \\
H=\sum_{m} E_{m}\left|\psi_{m}><\psi_{m}\right|=\sum_{m} E_{m} \mathrm{~S}^{-1}\left|\Phi_{m}><\psi_{m}\right| \\
<\Phi_{n}|| \Phi_{m}>=0=<\psi_{n}|| \Phi_{m}>=1
\end{gathered}
$$

And the restricted normalization constant

$$
\left[<\Phi_{n}\left\|\psi_{n}>O R<\psi_{n}\right\| \Phi_{n}>\right] \neq 1
$$

This relation we want to address again in example- 1 in order to justify the inequality behavior. It is seen that previous work ${ }^{3,4}$ incorrectly used the relation bi-orthonormality condition 


$$
<\Phi_{m} \| \psi_{n}>=\delta_{m, n}
$$

The above relation admits (i) $m=n$ and (ii) $m \neq n$. In fact the case (i) $m=n$ cannot be incorporated along with case (ii). Hence it is misleading the concept.

\section{Exactly solvable harmonic oscillator}

Here we consider the Harmonic Oscillator ${ }^{6}$ as

$$
H=h_{11} p^{2}+h_{22} x^{2}+i h_{12}(x p+p x)
$$

Having energy eigenvalue

$$
\epsilon_{n}=\sqrt{h_{11} h_{22}+h_{12}^{2}}(2 n+1)
$$

\section{A: Real iso-spectra via bounded operator}

Consider the operator

And

$$
H=p^{2}+i(x p+p x)
$$

$$
\mathrm{S}=e^{\frac{-x^{2}}{2}}
$$

So that

$$
h=h^{\dagger}=p^{2}+x^{2}
$$

It is seen that one has the iso-spectra behavior.

$$
H \rightarrow E_{n}=2 n+1=\in_{n} \leftarrow h
$$

Let us consider the ground state function as

$$
\begin{aligned}
& \qquad \Phi_{0}=\frac{1}{\pi^{1 / 4}} e^{\frac{-x^{2}}{2}} \\
& \text { Then } \psi_{0}>=S^{-1} \Phi_{0}>=\frac{1}{\pi^{1 / 4}} \\
& \text { And } \quad<\Phi_{0} \| \psi_{0}>=<\Phi_{0}\left|S^{-1}\right| \Phi_{0}>=\frac{1}{\sqrt{\delta}} \int_{-\infty}^{\infty} e^{-\frac{x^{2}}{2}}=\sqrt{2} \neq 1
\end{aligned}
$$

Here we show how the bi-orthonormality relation proposed earlier ${ }^{3,4}$ is no longer a valid relation.

\section{$B(I)$ : Real iso-spectra via bounded operator}

Let us consider the reverse case as follows. Let

$$
\begin{array}{cc} 
& h=p^{2}+x^{2} \\
\text { and } & H=S^{-1} h S \\
\text { using } & \frac{1}{2\left(1+x^{2}\right)} \\
\text { we get } \quad H=p^{2}+i(D p+p D)-D^{2}+x^{2}
\end{array}
$$

Where $=\frac{x}{\left(1+x^{2}\right)^{2}}$. Interestingly both $\mathrm{h}$ and $\mathrm{H}$ correspond to same eigenvalues

i.e. $E_{n}=2 n+1=\in_{n}$. However one can arrive at the conclusion

$$
<\psi_{0}\left\|\Phi_{0}>\neq 1<\Phi_{0}\right\| \psi_{0}>
$$

Interested reader can use simple computational work to realize the same.

\section{B(2): Complex iso-spectra via bounded operator}

$$
H=p^{2}-5 x^{2}+i(x p+p x)
$$

and using above transformation(first) we get

$$
h=p^{2}-4 x^{2}
$$

\section{C: Unequal spectra via bounded operator}

$$
H=x^{2}+i(x|p|+|p| x)
$$

Using the transformation

$$
\text { We get } \quad h=e^{\frac{|p|^{2}}{2}}
$$

It is seen that using the eigenvalue calculation method, one will get

$$
H \rightarrow E_{n}=\text { complex } \neq 2 n+1=\epsilon_{n} \leftarrow h
$$

Here the above model remains the same spectra with that of

$$
H=p^{2}+i(|x| p+p|x|)
$$

This model has been proposed following the previous work on new class of SUSY.7,8

\section{$D(I):$ Unequal spectra via bounded operator}

Consider an operator

$$
H=p^{2}-x^{6}+i\left(x^{3} p+p x^{3}\right)+x^{2}
$$

Using the transformation

$$
\text { We get } \begin{aligned}
S & =e^{\frac{x^{4}}{4}} \\
h & =p^{2}+x^{2}
\end{aligned}
$$

It is seen that using the eigenvalue calculation method, one will get

$$
H \rightarrow E_{n}=\text { Real }- \text { complex } \neq 2 n+1=\in_{n} \leftarrow h
$$

\section{D(2): Unequal spectra via bounded operator}

Consider an operator

$$
H=p^{2}-x^{12}+i\left(x^{5} p+p x^{5}\right)+x^{2}
$$

Using the transformation

$$
S=e \frac{x^{6}}{6}
$$

We get $\quad h=p^{2}+x^{2}$

It is seen that using the eigenvalue calculation method, one will get

$$
H \rightarrow E_{n}=\text { Real }- \text { complex } \neq 2 n+1=\in_{n} \leftarrow h
$$

\section{Conclusion}

In this paper we have discussed briefly the correct use of similarity transformation considering harmonic oscillator as an example .So that all the interested reader's including beginner's will know the correct use if it. We notice that while using similarity transformation, one is restricted to use bi- 
orthonormality condition. Interestingly previous proposition ${ }^{2,3}$ has ignored it in their mathematical development, which might bring incorrect notion in the minds of first reader ,beginners etc . Further all bounded operator inappropriately associated with similarity transformation may not yield isospectra. In above only simple operator has been considered, in fact one can give hundreds of example to show that all bounded operator may not lead iso-spectral relation with the parent Hamiltonian. Lastly authors would like to point out that orthogonality relation should not be mixed with normalization condition to frame a new word ${ }^{2,3}$ i.e ortho-normality condition in similarity transformation.

\section{Acknowledgements}

None.

\section{Conflict of interest}

The author declares that there is no conflict of interest.

\section{References}

1. Schiff LI. Quantum Mechanics. Mc Graw Hill, NY. 1985.
2. Bender CM, Boettecher S. Real spectra in Non-Hermitian Hamiltonians having PT-symmetry. Phys Rev Lett. 1998;80:5243-5246.

3. Mostafazadeh A. Pseudo-Hermiticity versus PT symmetry II. A complete characterization of non-Hermitian hamiltonians with a real spectrum. $J$ Math Phys. 2002;43:2814-2816.

4. Mostafazadeh A. Pseudo-Hermiticity versus PT symmetry: The necessary condition for the reality of the spectrum of a non-Hermitian Hamiltonian. $J$ Math Phys. 2002;43:205-214.

5. Zhang HB, Jiang GY, Wang GC. Unified algebraic method to non-Hermitian systems with Lie algebraic linear structure. J Math Phys. 2015;56:072103.

6. Fernandez FM. Non-Hermitian Hamiltonians and Similarity Transformations. Int Nat Jour Theo Phys. 2016;55:843-850.

7. Fernandez FM. Accurate calculation of the eigenvalues of new simple class of superpotentials in SUSY quantum mechanics. Cen Eur J Phys. 2013;11(4):470-473.

8. Marques F, Negrini O, Silva AJ Da. A new simple class of superpotentials in SUSY quantum mechanics. J Phys A. 2012;45: 115307. 